SCIENTIFIC PROCEEDINGS 2011, Faculty of Mechanical Engineering, STU in Bratislava Vol. 19, 2011, pp. 61-68, DOI: 10.2478/v10228-011-0011-2

\title{
APPROXIMATE MODEL OF ZONE SEDIMENTATION
}

\section{Ing. František DZIANIK, PhD.}

Institute of Process and Fluid Engineering, Faculty of Mechanical Engineering, Slovak University of Technology in Bratislava, Nám. slobody 17, 81231 Bratislava, Slovak Republic, frantisek.dzianik@stuba.sk

\begin{abstract}
The process of zone sedimentation is affected by many factors that are not possible to express analytically. For this reason, the zone settling is evaluated in practice experimentally or by application of an empirical mathematical description of the process. The paper presents the development of approximate model of zone settling, i.e. the general function which should properly approximate the behaviour of the settling process within its entire range and at the various conditions. Furthermore, the specification of the model parameters by the regression analysis of settling test results is shown. The suitability of the model is reviewed by graphical dependencies and by statistical coefficients of correlation. The approximate model could by also useful on the simplification of process design of continual settling tanks and thickeners.
\end{abstract}

KEY WORDS: zone sedimentation, approximate model, settling test, suspension thickening, settling curve, solids flux density

\section{INTRODUCTION}

The sedimentation in practice is relatively often applied to hydro-mechanical separating processes. It is employed, for instance, in waste water treatment for the thickening of industrial sludge, in ore treatment, and also in the gases de-dusting, in the size classification of solid particles etc. If the heterogeneous fluid mixtures contain a very low proportion of solid particles (less than $0,5 \%$ vol.), the interaction forces within the particles are negligible and the sedimentation of the particles in the gravitational field are possible to characterise with sufficient accuracy as unaffected $[3,6,7]$. At higher concentrations of particles in the mixtures, the additional force effects on the dispersed particles begin to appear more significantly. These force effects influence the settling movement of the particles. The sedimentation is considered as hindered in this case. The particles retain their individual nature, however, at the description of the sedimentation process it is necessary to assume the additional effects on sedimentation $[3,6,7]$.

If the concentration of particles in a mixture is so high, that particles lose their individual nature and the mixture behaves in the gravitational field as a porous layer with variable concentration over time and also along the height of the layer, the sedimentation is considered as gravitational thickening of concentrated heterogeneous mixture, usually in suspension. Such processes are called zone sedimentation. It is characterised by the progressive displacement of liquid out of the pores of concentrated suspension which is proven by the progressive sinking of the interface between the layer of concentrated suspension and the formatting layer of clarified liquid. In this case, the velocity of sedimentation is considered exactly by the sinking velocity of that interface. The behaviour of the batch zone sedimentation is represented usually graphically, by a so-called settling curve. During this process, the four characteristic regions - zones are generally formed (Fig. 1). A typical behaviour of the settling curve is depicted in Fig. 1. The settling curves are usually obtained by the settling tests which are performed in glass cylinders with the actual 
suspension. The settling curves are the important basis for the proposition of industrial settling units.

There are a lot of scientific and technical publications that deal with the process of zone sedimentation from various aspects and purposes. However, the general analytical description of the process is not possible to define in regard to many factors by which it is affected. For this reason, the various empirical or semi-empirical approximate models are developed in order to describe that process at least partially. The validity of these models is usually limited only for certain phases and certain conditions of the process. An example could be the paper [2] that presents the overview of some published models as well as the development of another model of activated sludge settling. Furthermore, paper [1] describes the development of the mathematical model in which the influence of initial suspension concentration on the sedimentation behaviour of certain types of suspensions is included. The work [8] deals with the mathematical description of the settling process which consider the flocculation process during zone sedimentation.

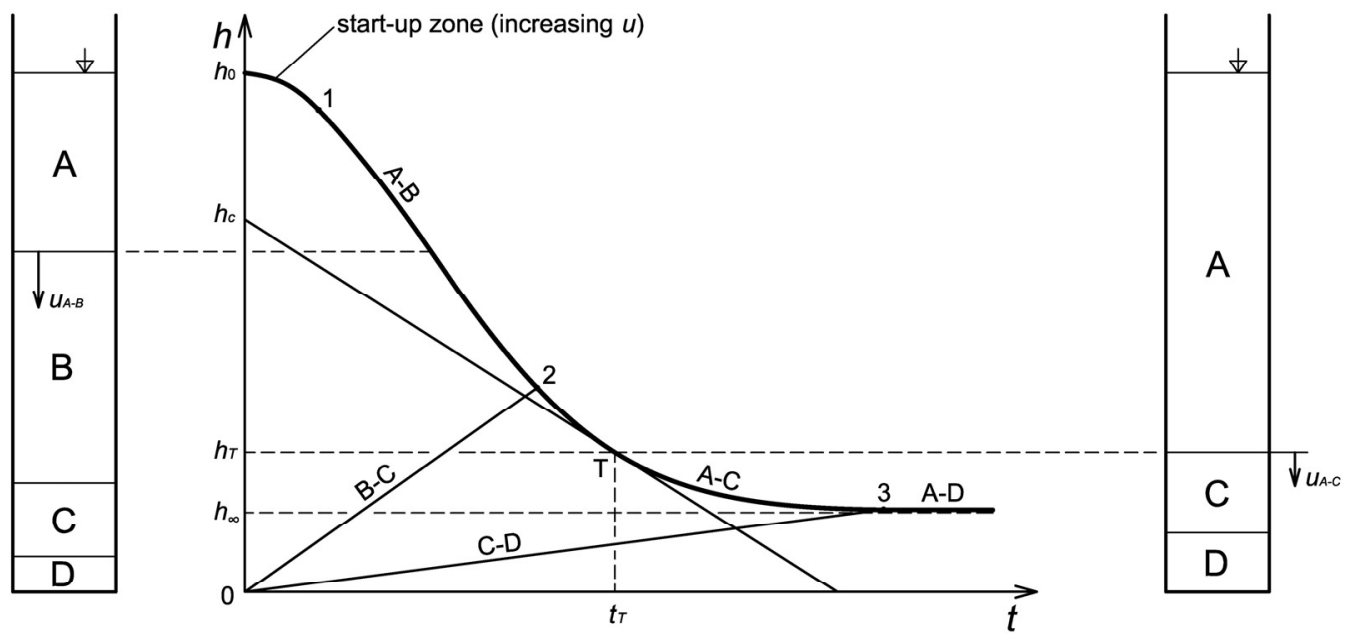

Fig. 1 Typical zone sedimentation process - the settling curve $A-$ clarified supernatant zone $(c \rightarrow 0)$,

$B$ - zone of settling suspension with approximately constant concentration $c$, $C$-zone of concentrated suspension with downward increasing concentration $c$,

$$
D \text {-sludge zone }\left(c \rightarrow c_{\max }\right) \text {. }
$$

\section{THE PROPOSITION OF AN APPROXIMATE MATHEMATICAL DESCRIPTION OF THE ZONE SEDIMENTATION PROCESS}

An approximate mathematical model is the function that approximates, optimally, the real behaviour of dependency between the values. It is characterised by the appropriate properties in terms of potential variability of approximated dependencies to be general for the given purpose. The approximate model includes the parameters that are necessary to be specified by a proper method. Usually the results of the experimental measurements are utilised for this purpose. From their range, it follows also the validity range of the model with its specific parameters. In this case, the aim is to propose the approximate model of zone settling, i.e. the general function which should properly approximate the experimentally acquired behaviour of various settling curves. 
SCIENTIFIC PROCEEDINGS 2011, Faculty of Mechanical Engineering, STU in Bratislava Vol. 19, 2011, pp. 61-68, DOI: 10.2478/v10228-011-0011-2

Furthermore, specification of the model parameters for a concrete case will be shown and also review of the suitability of the model by statistics.

Several publications $[1,3,7]$ present differential equations for the kinetic expression of the partition of zone settling process, given by:

$$
\frac{\mathrm{d} h}{\mathrm{~d} t}=-k\left(h-h_{\infty}\right)
$$

wherein $h$ is the level of the interface between suspension and supernatant liquid, $t$ is the time, $h_{\infty}$ is the value of $h$ when $t \rightarrow \infty$ and $k$ is the parameter called the rate constant. The equation (1) relatively well describes the behaviour of the partition of zone settling process when the interface between suspension and supernatant liquid occurs in transition zone and compression zone (the zones that are characterised by the progressive increasing of local concentration of suspension at the interface and by the considerable deceleration of its movement).

The imperfection of equation (1) is its limited validity for a range of transition zone and compression zone. This imperfection eliminates the proposition of general function which would approximate properly whole the behaviour of a settling curve, including relatively short start-up zone when the velocity of suspension-supernatant interface movement increases, unlike the function acquired by solution of the equation (1). Such function is possible to acquire by the solution of adapted differential equation:

$$
\frac{\mathrm{d} h}{\mathrm{~d} t}=-F(t)\left(h-h_{\infty}\right) .
$$

Equation (2) includes the rate function $F(t)$ that is expressed by the relation

$$
F=p m t^{m-1}
$$

wherein $p$ and $m$ are the parameters. By the substitution of equation (3) into the equation (2), the differential equation

$$
\frac{\mathrm{d} h}{h-h_{\infty}}=-p m t^{m-1} \mathrm{~d} t
$$

is acquired. The general solution of equation (4) has a form

$$
h=h_{\infty}+\left(h_{0}-h_{\infty}\right) \exp \left(-p t^{m}\right)
$$

and has been obtained by the help of time and boundary conditions which define the process of batch zone settling. At the beginning of the process $(t=0 \mathrm{~s})$, the suspension-supernatant interface level and the surface level of batch $h_{0}$ are identical $\left(h=h_{0}\right)$. After the sufficiently long time (theoretically infinite time $t=\infty$ ), the interface level $h$ corresponds to the thickness of sludge layer $\left(h=h_{\infty}\right)$. The function $h=\mathrm{f}(t)$, that is given by equation (5), is possible to express in form

$$
\Delta h^{*}=\exp \left(-p t^{m}\right)
$$

where $\Delta h^{*}$ is dimensionless level of interface between suspension and supernatant liquid: 
SCIENTIFIC PROCEEDINGS 2011, Faculty of Mechanical Engineering, STU in Bratislava Vol. 19, 2011, pp. 61-68, DOI: 10.2478/v10228-011-0011-2

$$
\Delta h^{*}=\frac{h-h_{\infty}}{h_{0}-h_{\infty}} .
$$

In order to employ exactly the laboratory settling tests for the proposition of industrial settling units, the values of the instantaneous concentration $c$ at the suspension-supernatant interface and its instantaneous movement velocities $u$ are necessary to determine. The movement velocities of interface $u$ and corresponding suspension concentration at the interface $c$ define the value of solids flux density $\Psi$ :

$$
\Psi=u c .
$$

The necessary settling area $A$ of the settling tanks is possible to specify by the solids flux density $\Psi$ if certain capacity and certain thickening degree of suspension are required $[3,4,6,7,9]$. The overall minimal achieved value of solids flux density within the settling tanks in the settling direction is important for the specification of settling area largeness. Such value of the solids flux density is also called the critical $\Psi_{c k}$ and is defined by equation

$$
\Psi_{c k}=\frac{\dot{V}_{s} c_{s}}{A}=\frac{\dot{V}_{k} c_{k}}{A},
$$

where in $A$ is necessary minimal largeness of the settling area in order to achieve the total clarification of the supernatant liquid if the increase of suspension concentration from the value $c_{s}$ to value $c_{k}$ is required and if $\dot{V}_{s}$ is the volumetric flow rate of suspension into the tank and $\dot{V}_{k}$ is the volumetric flow rate of thickened sludge out of the tank. The overall solids flux density $\Psi_{c}$ within the continual settling tank is given by the relation

$$
\Psi_{c}=\Psi+\Psi_{0},
$$

where in $\Psi$ is the solids flux density due to the sedimentation within the tank (equation 8) and $\Psi_{0}$ is the solids flux density caused by the continuous outflow of the thickened sludge out of the tank.

The instantaneous velocity $u$ of the suspension-supernatant interface movement at a settling test is given by the equation (2). Its expression by the relation

$$
u=\frac{\mathrm{d} h}{\mathrm{~d} t}=-p m t^{m-1}\left(h_{0}-h_{\infty}\right) \exp \left(-p t^{m}\right)
$$

is obtained by the substitution of the rate function $F(t)$ (equation 3 ) and the instantaneous interface level (equation 5) into the equation (2). The instantaneous suspension concentration $c$ at the interface is essentially possible to calculate on the basis of Kynch theory which is in more detail mentioned in the publications [3, 5, 6]. However, the Kynch theory is not possible to generalise for the settling analysis of real suspensions because in most cases the assumptions of this theory are not fulfilled. Nevertheless, the instantaneous concentration of suspension $c$ at the interface between suspension and supernatant liquid will be assess according to the relation

$$
c=c_{0} \frac{h_{0}}{h_{c}}
$$


which follows from the Kynch theory of sedimentation for the occurrence of suspensionsupernatant interface level in the transition zone or in the compression zone $[3,5,6]$. The value $h_{c}$ is the coordinate of intersection point with the $h$-axis of the tangent to settling curve (Fig. 1 ) and $c_{0}$ is the initial suspension concentration. From the mathematical point of view, the relation (12) is possible to apply within the entire process of batch zone sedimentation, however with the recommendation of experimental verification. The equation of tangent to the settling curve has a general expression:

$$
h=\frac{\mathrm{d} h}{\mathrm{~d} t} t+h_{c} .
$$

The equation (13), after the substitution of equation (11) and its modification, acquires the form:

$$
h=-\left(h_{0}-h_{\infty}\right) p m t^{m} \exp \left(-p t^{m}\right)+h_{c}
$$

from which the relation for $h_{c}$ calculation, after the substitution of equation (5) for $h$, follows:

$$
h_{c}=\left(h_{0}-h_{\infty}\right)\left(1+p m t^{m}\right) \exp \left(-p t^{m}\right)+h_{\infty} .
$$

Then it is possible, considering the equation (12), to obtain the relation for the instantaneous concentration $c$ of suspension at the suspension-supernatant interface:

$$
c=\frac{c_{0} h_{0}}{\left(h_{0}-h_{\infty}\right)\left(1+p m t^{m}\right) \exp \left(-p t^{m}\right)+h_{\infty}} .
$$

The following expression of solids flux density function $\Psi$ for batch zone sedimentation has been derived on the basis of equations (8), (11) and (16):

$$
\Psi=\frac{-p m t^{m-1}\left(h_{0}-h_{\infty}\right) \exp \left(-p t^{m}\right) c_{0} h_{0}}{\left(h_{0}-h_{\infty}\right)\left(1+p m t^{m}\right) \exp \left(-p t^{m}\right)+h_{\infty}} .
$$

\section{EXPERIMENTAL VERIFICATION OF DEVELOPED MATHEMATICAL MODEL}

The suitability of developed approximate empirical model of zone sedimentation has been verified by laboratory settling tests. The tests were performed in glass cylinders. As a tested material, the water suspension of lime hydrate with three initial concentrations $c_{0}=0,5 ; 3$ and $5 \%$ vol. was used. The size of particles in suspension was up to $10 \mu \mathrm{m}$ and their density $2240 \mathrm{~kg} \cdot \mathrm{m}^{-3}$.

The tests results are presented graphically in form of function dependencies $h=\mathrm{f}(t)$ of suspension-supernatant interface height $h$ on settling time $t$ (Fig. 2). The measured values are plotted in Fig. 2 as the points. The continuous functions of the settling curves are also included in Fig. 2. These have been acquired by non-linear regression according to proposed empirical model (equation 5) from the measured values. Acquired values of model parameters for individual settling tests are listed in Table 1. The suitability of proposed approximate model is possible to review by the correlation graph depicted in Fig. 3 and by the corresponding coefficients of correlation $R^{2}$. 
SCIENTIFIC PROCEEDINGS 2011, Faculty of Mechanical Engineering, STU in Bratislava Vol. 19, 2011, pp. 61-68, DOI: 10.2478/v10228-011-0011-2

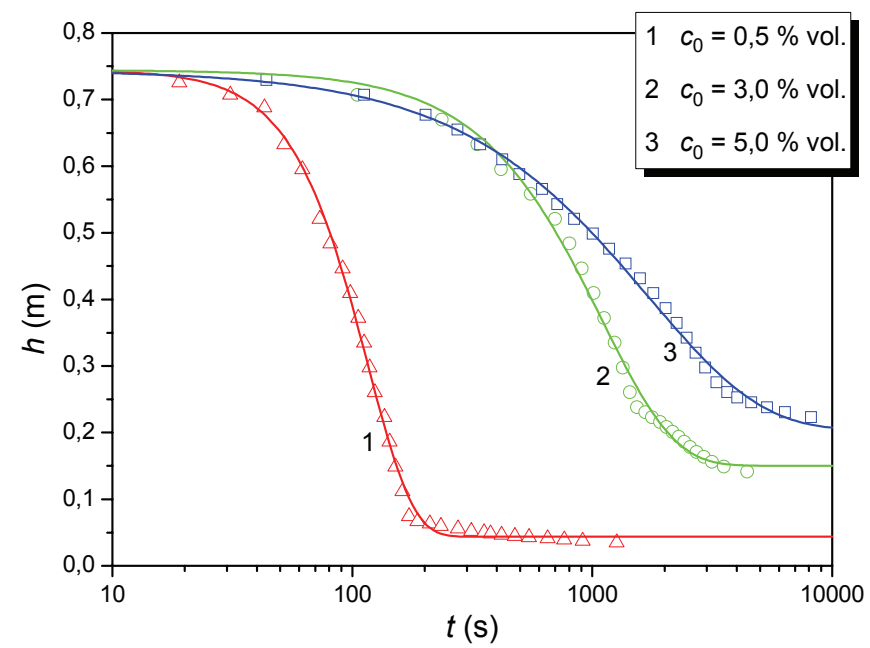

Fig. 2 The settling curves $h=f(t)$ at various initial concentrations of suspension $c_{0}$ - measured values and the functions of proposed mathematical model.

\begin{tabular}{|c|c|c|c|c|c|c|}
\hline $\begin{array}{c}\text { test } \\
\text { number }\end{array}$ & $\begin{array}{c}c_{0} \\
(-)\end{array}$ & $\begin{array}{c}h_{0} \\
(\mathrm{~m})\end{array}$ & $\begin{array}{c}h_{\infty} \\
(\mathrm{m})\end{array}$ & $\begin{array}{c}p \cdot 10^{2} \\
\left(\mathrm{~s}^{-\mathrm{m}}\right)\end{array}$ & $\begin{array}{c}m \\
(-)\end{array}$ & $\begin{array}{c}R^{2} \\
(-)\end{array}$ \\
\hline 1 & 0,005 & 0,744 & 0,044 & 0,861 & 2,316 & 0,998 \\
2 & 0,030 & 0,744 & 0,150 & 0,091 & 1,442 & 0,995 \\
3 & 0,050 & 0,744 & 0,204 & 0,058 & 0,926 & 0,997 \\
\hline
\end{tabular}

Tab. 1 The parameters values of mathematical model.

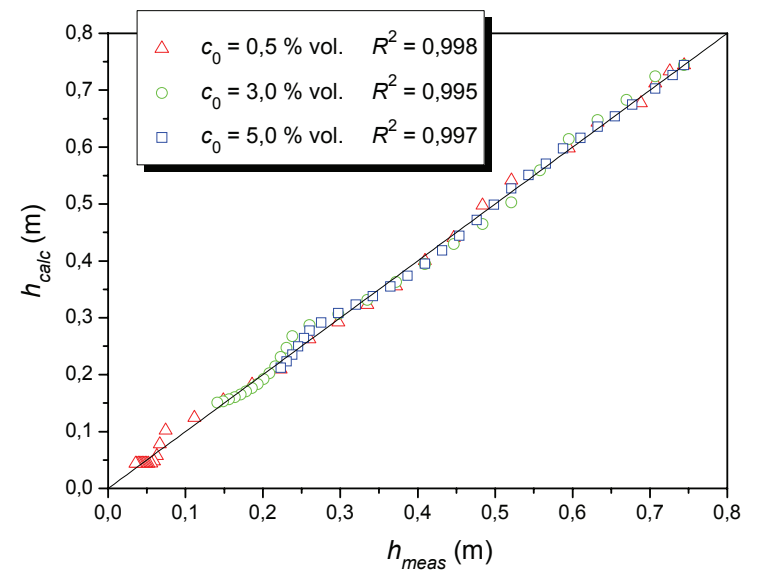

Fig. 3 The correlation dependence between measured values of the interface height $h_{\text {meas }}$ and calculated according to mathematical model $h_{\text {calc. }}$.

Fig. 4 presents the calculated dependencies of instantaneous concentration $c$ at the interface between suspension and supernatant liquid on the settling time $t$. Points in Fig. 4 have 
SCIENTIFIC PROCEEDINGS 2011, Faculty of Mechanical Engineering, STU in Bratislava Vol. 19, 2011, pp. 61-68, DOI: 10.2478/v10228-011-0011-2

been calculated according to equation (16) that follows from the proposed model. They correspond to time values of measured points in settling curves in Fig. 2. In a similar way, there are presented in Fig. 5 calculated dependencies of solids flux density $\Psi$ on concentration $c$ at the interface between suspension and supernatant liquid. The negative values of solids flux density follow from the negative change of suspension-supernatant interface height in time - the interface is sinking in time.

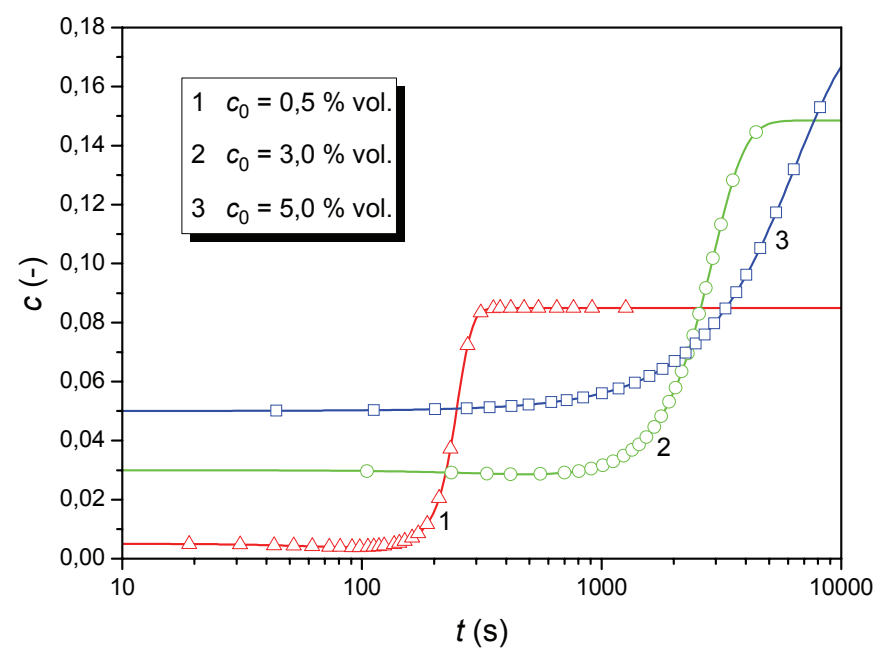

Fig. 4 Calculated dependencies of instantaneous concentration c at the interface between suspension and supernatant liquid on the settling time t at various initial concentrations of suspension $c_{0}$.

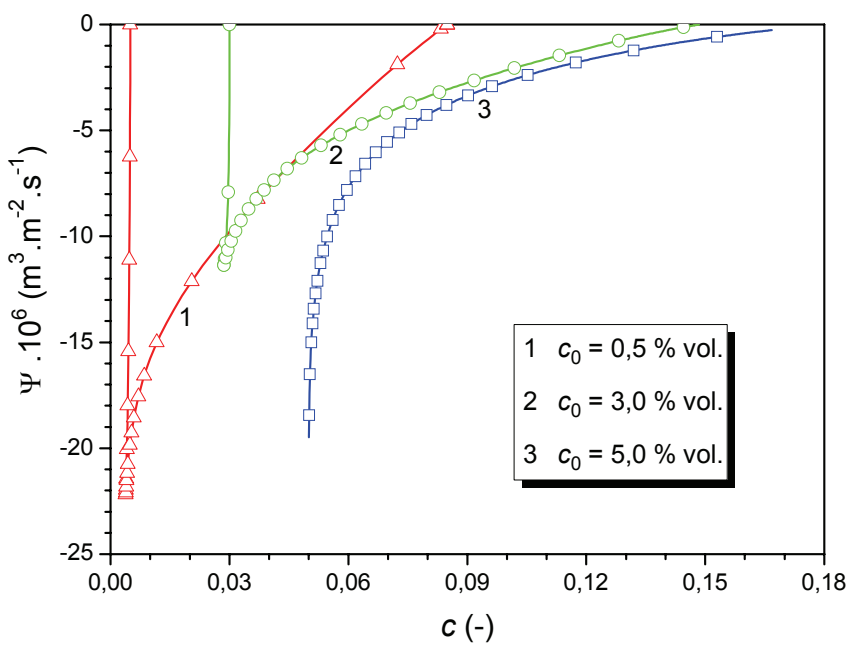

Fig. 5 Calculated dependencies of solids flux density $\Psi$ on concentration $c$ at the interface between suspension and supernatant liquid at various initial concentrations of suspension $c_{0}$.

\section{CONCLUSION}


The paper presents the development and application of the approximate empirical model for the general description of batch zone sedimentation. The suitability of the model has so far been verified by several settling tests with fine suspension in relatively wide range of concentrations. Good suitability of the proposed model follows from the obtained results. For more general conclusions, it is necessary to perform more experiments with more types of suspensions. On the basis of these experiments it would be possible to specify the parameters of the model as functions of some suspension properties - initial concentration of suspension $c_{0}$, mean particles size, polydispersity index, particles density, liquid density etc. the expression of the model in nondimensional form is proposed for future works to achieve a larger generalization. The approximate mathematical model of the batch zone sedimentation also makes the proposition of continual settling tanks or thickeners easier according to published methodology in $[3,4,6,7,9]$.

\section{REFERENCES}

[1] BHARGAVA, D. S., RAJAGOPAL, K.: Differentiation between transition zone and compression in zone settling. Water Research, Vol. 27, No. 3, 1993, pp. 457-463.

[2] CHO, S. H., COLIN, F., SARDIN, M., PROST, C.: Settling velocity model of activated sludge. Water Research, Vol. 27, No. 7, 1993, pp. 1237-1242.

[3] COULSON, J. M., RICHARDSON, J. F., HARKER, J. H., BACKHURST, J. R.: Chemical engineering. Volume 2. Particle technology and separation processes. Butterworth Heinemann, Oxford 2002.

[4] HASSETT, N. J.: Theories of the operation of continuous thickeners. Industrial Chemist, 37 , 1961, p. 25.

[5] KYNCH, G. J.: A theory of sedimentation. Journal of the Chemical Society: Faraday Transactions, 48, 1952, pp. 166-176.

[6] NOVÁK, V., RIEGER, F., VAVRO, K.: Hydraulické pochody v chemickém a potravinářském průmyslu. SNTL, Praha 1989.

[7] TUČEK, F., CHUDOBA, J., KONÍČEK, Z.: Základní procesy a výpočty v technologii vody. SNTL, Praha 1988

[8] VAN DEVENTER, B. B. G., USHER, S. P., KUMAR, A., RUDMAN, M., SCALES, P. J.: Aggregate densification and batch settling. Chemical Engineering Journal, 171, 2011, pp. 141151.

[9] YOSHIOKA, N., HOTTA, Y., TANAKA, S., NAITO, S., TSUGAMI, S.: Continuous thickening of homogeneous flocculated slurries. Kagaku Kogaku (J. Soc. Chem. Eng., Japan ), 21, 1957, pp. 66-74. 